\title{
The Teacher-Student Chatroom Corpus
}

\section{Andrew Caines ${ }^{1}$ Helen Yannakoudakis ${ }^{2}$ Helena Edmondson ${ }^{3}$ Helen Allen ${ }^{4}$ Pascual Pérez-Paredes ${ }^{5} \quad$ Bill Byrne $^{6} \quad$ Paula Buttery $^{1}$}

\author{
${ }^{1}$ ALTA Institute \& Computer Laboratory, University of Cambridge, U.K. \\ \{andrew.caines|paula.buttery\}@cl.cam.ac.uk \\ ${ }^{2}$ Department of Informatics, King's College London, U.K. \\ helen.yannakoudakis@kcl.ac.uk \\ ${ }^{3}$ Theoretical \& Applied Linguistics, University of Cambridge, U.K. \\ hle24 @ cantab.ac.uk \\ ${ }^{4}$ Cambridge Assessment, University of Cambridge, U.K. \\ allen.h@cambridgeenglish.org \\ ${ }^{5}$ Faculty of Education, University of Cambridge, U.K. \\ pfp23@cam.ac.uk \\ ${ }^{6}$ Department of Engineering, University of Cambridge, U.K. \\ bill.byrnedeng.cam.ac.uk
}

\begin{abstract}
The Teacher-Student Chatroom Corpus (TSCC) is a collection of written conversations captured during one-to-one lessons between teachers and learners of English. The lessons took place in an online chatroom and therefore involve more interactive, immediate and informal language than might be found in asynchronous exchanges such as email correspondence. The fact that the lessons were one-to-one means that the teacher was able to focus exclusively on the linguistic abilities and errors of the student, and to offer personalised exercises, scaffolding and correction. The TSCC contains more than one hundred lessons between two teachers and eight students, amounting to $13.5 \mathrm{~K}$ conversational turns and $133 \mathrm{~K}$ words: it is freely available for research use. We describe the corpus design, data collection procedure and annotations added to the text. We perform some preliminary descriptive analyses of the data and consider possible uses of the TSCC.
\end{abstract}

\section{Introduction \& Related Work}

We present a new corpus of written conversations from one-to-one, online lessons between English language teachers and learners of English. This

\footnotetext{
This work is licensed under a Creative Commons Attribution-NonCommercial-ShareAlike 4.0 International Licence. Licence details: http://creativecommons. org/licenses/by-nc-sa/4.0
}

is the Teacher-Student Chat Corpus (TSCC) and it is openly available for research use ${ }^{1}$. TSCC currently contains 102 lessons between 2 teachers and 8 students, which in total amounts to $13.5 \mathrm{~K}$ conversational turns and $133 \mathrm{~K}$ word tokens, and it will continue to grow if funding allows.

The corpus has been annotated with grammatical error corrections, as well as discourse and teaching-focused labels, and we describe some early insights gained from analysing the lesson transcriptions. We also envisage future use of the corpus to develop dialogue systems for language learning, and to gain a deeper understanding of the teaching and learning process in the acquisition of English as a second language.

We are not aware of any such existing corpus, hence we were motivated to collect one. To the best of our knowledge, the TSCC is the first to feature one-to-one online chatroom conversations between teachers and students in an English language learning context. There are of course many conversation corpora prepared with both close discourse analysis and machine learning in mind. For instance, the Cambridge and Nottingham Corpus of Discourse in English (CANCODE) contains spontaneous conversations recorded in a wide variety of informal settings and has been used to study the grammar of spoken interaction (Carter and McCarthy, 1997). Both versions 1 and 2 of

\footnotetext{
${ }^{1}$ Available for download from https: / forms.gle/ oW5fWTTZfZcTkp8v9
}

Andrew Caines, Helen Yannakoudakis, Helena Edmondson, Helen Allen, Pascual Pérez-Paredes, Bill Byrne and Paula Buttery 2020. The teacher-student chatroom corpus. Proceedings of the 9th Workshop on Natural Language Processing for Computer Assisted Language Learning (NLP4CALL 2020). Linköping Electronic Conference Proceedings 175: 10-9. 
the British National Corpus feature transcriptions of spoken conversation captured in settings ranging from parliamentary debates to casual discussion among friends and family (BNC Consortium, 2001; Love et al., 2017).

Corpora based on educational interactions, such as lectures and small group discussion, include the widely-used Michigan Corpus of Academic Spoken English (MICASE) (Simpson et al., 2002), TOEFL 2000 Spoken and Written Academic Language corpus (Biber et al., 2004), and Limerick Belfast Corpus of Academic Spoken English (LIBEL) (O'Keeffe and Walsh, 2012). Corpora like the ones listed so far, collected with demographic and linguistic information about the contributors, enable the study of sociolinguistic and discourse research questions such as the interplay between lexical bundles and discourse functions (Csomay, 2012), the interaction of roles and goal-driven behaviour in academic discourse (Evison, 2013), and knowledge development at different stages of higher education learning (Atwood et al., 2010).

On a larger scale, corpora such as the MultiDomain Wizard-of-Oz datasets (MultiWOZ) contain thousands of goal-directed dialogue collected through crowdsourcing and intended for the training of automated dialogue systems (Budzianowski et al., 2018; Eric et al., 2020). Other work has involved the collation of pre-existing conversations on the web, for example from Twitter (Ritter et al., 2010), Reddit (Schrading et al., 2015), and movie scripts (Danescu-Niculescu-Mizil and Lee, 2011). Such datasets are useful for training dialogue systems to respond to written inputs - so-called 'chatbots' - which in recent years have greatly improved in terms of presenting some kind of personality, empathy and world knowledge (Roller et al., 2020), where previously there had been relatively little of all three. The improvement in chatbots has caught the attention of, and in turn has been driven by, the technology industry, for they have clear commercial applications in customer service scenarios such as helplines and booking systems.

As well as personality, empathy and world knowledge, if chatbots could also assess the linguistic proficiency of a human interlocutor, give pedagogical feedback, select appropriate tasks and topics for discussion, maintain a long-term memory of student language development, and begin and close a lesson on time, that would be a teaching chatbot of sorts. We know that the list above represents a very demanding set of technological challenges, but the first step towards these more ambitious goals is to collect a dataset which allows us to analyse how language teachers operate, how they respond to student needs and structure a lesson. This dataset may indicate how we can begin to address the challenge of implementing a language teaching chatbot.

We therefore set out to collect a corpus of oneto-one teacher-student language lessons in English, since we are unaware of existing corpora of this type. The most similar corpora we know of are the Why2Atlas Human-Human Typed Tutoring Corpus which centred on physics tutoring (Rosé et al., 2003), the chats collected between native speakers and learners of Japanese using a virtual reality university campus (Toyoda and Harrison, 2002), and an instant messaging corpus between native speakers and learners of German (Höhn, 2017). This last corpus was used to design an other-initiated self-repair module in a dialogue system for language learning, the kind of approach which we aim to emulate in this project. In addition there is the CIMA dataset released this year which involves one-to-one written conversation between crowdworkers role-playing as teachers and students (Stasaski et al., 2020), but the fact that they are not genuinely teachers and learners of English taking part in real language lessons means that the data lack authenticity (albeit the corpus is well structured and useful for chatbot development).

\section{Corpus design}

We set out a design for the TSCC which was intended to be convenient for participants, efficient for data processing, and would allow us to make the data public. The corpus was to be teachercentric: we wanted to discover how teachers deliver an English language lesson, adapt to the individual student, and offer teaching feedback to help students improve. On the other hand, we wanted as much diversity in the student group as possible, and therefore aimed to retain teachers during the data collection process as far as possible, but to open up student recruitment as widely as possible.

In order to host the lessons, we considered several well-known existing platforms, including Facebook Messenger, WhatsApp, and Telegram, but decided against these due firstly to concerns about connecting people unknown to each other, 
where the effect of connecting them could be longlasting and unwanted (i.e. ongoing messaging or social networking beyond the scope of the TSCC project). Secondly we had concerns that since those platforms retain user data to greater or lesser extent, we were requiring that study participants give up some personal information to third party tech firms - which they may already be doing, but we didn't want to require this of the participants.

We consequently decided to use ephemeral chatrooms to host the lessons, and looked into using existing platforms such as Chatzy, but again had privacy concerns about the platform provider retaining their own copy of the lesson transcriptions (a stated clause in their terms and conditions) for unknown purposes. Thus we were led to developing our own chatroom in Shiny for R (Chang et al., 2020). In designing the chatroom we kept it as minimal and uncluttered as possible; it had little extra functionality but did the basics of text entry, username changes, and link highlighting.

Before recruiting participants, we obtained ethics approval from our institutional review board, on the understanding that lesson transcripts would be anonymised before public release, that participant information forms would be at an appropriate linguistic level for intermediate learners of English, and that there would be a clear procedure for participants to request deletion of their data if they wished to withdraw from the study. Funding was obtained in order to pay teachers for their participation in the study, whereas students were not paid for participation on the grounds that they were receiving a free one-to-one lesson.

\section{Data collection}

We recruited two experienced, qualified English language teachers to deliver the online lessons one hour at a time, on a one-to-one basis with students. The teacher-student pair were given access to the chatroom web application (Figure 1) and we obtained a transcription of the lesson at the end of the lesson.

When signing up to take part in the study, all participants were informed that the contents of the lesson would be made available to researchers in an anonymised way, but to avoid divulging personally identifying information, or other information they did not wish to be made public. A reminder to this effect was displayed at the start of every chatroom lesson. As an extra precaution, we made anonymisation one part of the transcription annotation procedure; see the next section for further detail.

Eight students have so far been recruited to take part in the chatroom English lessons which form this corpus. All students participated in at least 2 lessons each $(\max =32$, mean $=12)$. Therefore one possible use of the corpus is to study longitudinal pedagogical effects and development of second language proficiency in written English chat. At the time of data collection, the students were aged 12 to 40, with a mean of 23 years. Their first languages are Japanese (2), Ukrainian (2), Italian, Mandarin Chinese, Spanish, and Thai.

We considered being prescriptive about the format of the one-hour lessons, but in the end decided to allow the teachers to use their teaching experience and expertise to guide the content and planning of lessons. This was an extra way of discovering how teachers structure lessons and respond to individual needs, while also observing what additional resources they call on (other websites, images, source texts, etc). When signing up to participate in the study, the students were able to express their preferences for topics and skills to focus on, information which was passed on to the teachers in order that they could prepare lesson content accordingly. Since most students return for several lessons with the teachers, we can also observe how the teachers guide them through the unwritten 'curriculum' of learning English, and how students respond to this long-term treatment.

\section{Annotation}

The 102 collected lesson transcriptions have been annotated by an experienced teacher and examiner of English. The transcriptions were presented as spreadsheets, with each turn of the conversation as a new row, and columns for annotation values. There were several steps to the annotation process, listed and described below.

Anonymisation: As a first step before any further annotation was performed, we replaced personal names with $\langle$ TEACHER $\rangle$ or $\langle$ STUDENT $\rangle$ placeholders as appropriate to protect the privacy of teacher and student participants. For the same reason we replaced other sensitive data such as a date-of-birth, address, telephone number or email address with $\langle\mathrm{DOB}\rangle,\langle$ ADDRESS $\rangle$, $\langle$ TELEPHONE $\rangle,\langle$ EMAIL $\rangle$. Finally, any personally identifying information - the mention of a place of 


\section{ShinyChat}

For teacher-student conversations

Welcome to the ShinyChatRoom! Maintained by researchers at the University of Cambridge. By chatting here you agree that these conversations will be recorded and used for research. For help please contact chat.corpus@cl.cam.ac.uk

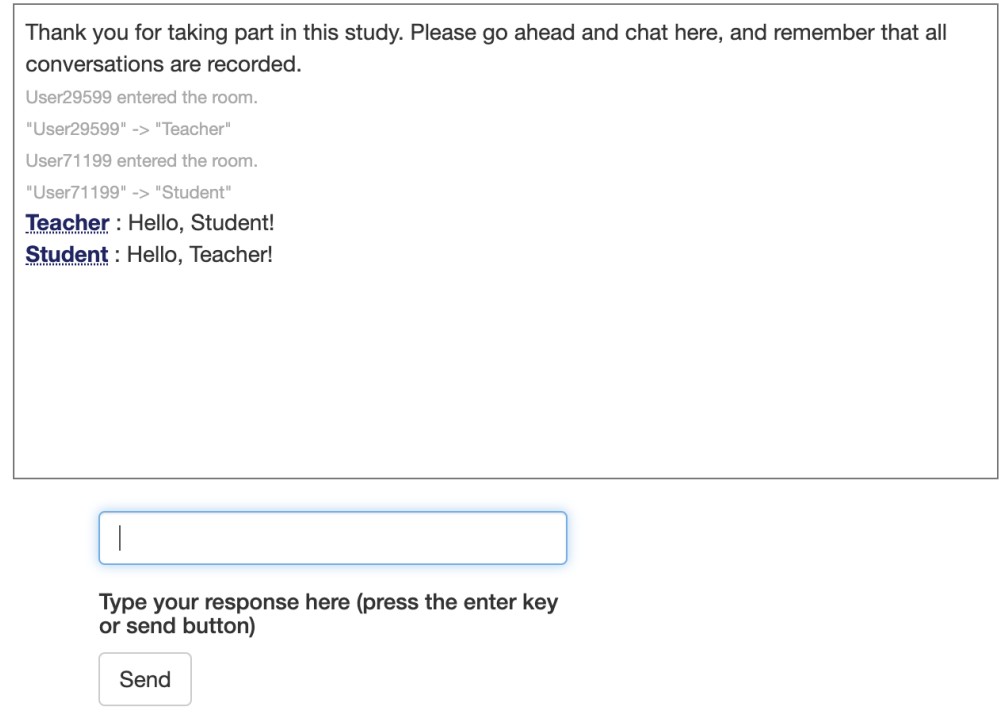

Please set your username here:

Teacher

Connected Users

- Teacher

- Student

Figure 1: Screenshot of the 'ShinyChat' chatroom

work or study, description of a regular pattern of behaviour, etc - was removed if necessary.

Grammatical error correction: As well as the original turns of each participant, we also provide grammatically corrected versions of the student turns. The teachers make errors too, which is interesting in itself, but the focus of teaching is on the students and therefore we economise effort by correcting student turns only. The process includes grammatical errors, typos, and improvements to lexical choice. This was done in a minimal fashion to stay as close to the original meaning as possible. In addition, there can often be many possible corrections for any one grammatical error, a known problem in corpus annotation and NLP work on grammatical errors (Bryant and $\mathrm{Ng}, 2015$ ). The usual solution is to collect multiple annotations, which we have not yet done, but plan to. In the meantime, the error annotation is useful for grammatical error detection even if correction might be improved by more annotation.

Responding to: This step involves the disentangling of conversational turns so that it was clear which preceding turn was being addressed, if it was not the previous one. As will be familiar from messaging scenarios, people can have conversations in non-linear ways, sometimes referring back to a turn long before the present one. For example, the teacher might write something in turn number 1 , then something else in turn 2. In turn 3 the student responds to turn 2 - the previous one, and therefore an unmarked occurrence - but in turn 4 they respond to turn 1 . The conversation 'adjacency pairs' are thus non-linear, being $1 \& 4,2 \& 3$.

Sequence type: We indicate major and minor shifts in conversational sequences - sections of interaction with a particular purpose, even if that purpose is from time-to-time more social than it is educational. Borrowing key concepts from the CONVERSATION ANALYSIS (CA) approach (Sacks et al., 1974), we seek out groups of turns which together represent the building blocks of the chat transcript: teaching actions which build the structure of the lessons.

CA practitioners aim 'to discover how participants understand and respond to one another in their turns at talk, with a central focus on how sequences of action are generated' (Seedhouse (2004) quoting Hutchby and Wooffitt (1988), 
emphasis added).

We define a number of sequence types listed and described below, firstly the major and then the minor types, or 'sub-sequences':

- Opening - greetings at the start of a conversation; may also be found mid-transcript, if for example the conversation was interrupted and conversation needs to recommence.

- Topic (minor labels complete this sequence type).

- Exercise - signalling the start of a constrained language exercise (e.g. 'please look at textbook page 50', 'let's look at the graph', etc); can be controlled or freer practice (e.g. gap-filling versus prompted re-use).

- Redirection - managing the conversation flow to switch from one topic or task to another.

- Disruption - interruption to the flow of conversation for some reason; for example because of loss of internet connectivity, telephone call, a cat stepping across the keyboard, and so on...

- Homework - the setting of homework for the next lesson, usually near the end of the present lesson.

- Closing - appropriate linguistic exchange to signal the end of a conversation.

Below we list our minor sequence types, which complement the major sequence types:

- Topic opening - starting a new topic: will usually be a new sequence.

- Topic development - developing the current topic: will usually be a new subsequence.

- Topic closure - a sub-sequence which brings the current topic to a close.

- Presentation - (usually the teacher) presenting or explaining a linguistic skill or knowledge component.

- Eliciting - (usually the teacher) continuing to seek out a particular response or realisation by the student.

- Scaffolding - (usually the teacher) giving helpful support to the student.

- Enquiry - asking for information about a specific skill or knowledge component.
- Repair - correction of a previous linguistic sequence, usually in a previous turn, but could be within a turn; could be correction of self or other.

- Clarification - making a previous turn clearer for the other person, as opposed to 'repair' which involves correction of mistakes.

- Reference - reference to an external source, for instance recommending a textbook or website as a useful resource.

- Recap - (usually the teacher) summarising a take-home message from the preceding turns.

- Revision - (usually the teacher) revisiting a topic or task from a previous lesson.

Some of these sequence types are exemplified in Table 1.

Teaching focus: Here we note what type of knowledge is being targeted in the new conversation sequence or sub-sequence. These usually accompany the sequence types, Exercise, Presentation, Eliciting, Scaffolding, Enquiry, Repair and Revision.

- Grammatical resource - appropriate use of grammar.

- Lexical resource - appropriate and varied use of vocabulary.

- Meaning - what words and phrases mean (in specific contexts).

- Discourse management - how to be coherent and cohesive, refer to given information and introduce new information appropriately, signal discourse shifts, disagreement, and so on.

- Register - information about use of language which is appropriate for the setting, such as levels of formality, use of slang or profanity, or intercultural issues.

- Task achievement - responding to the prompt in a manner which fully meets requirements.

- Interactive communication - how to structure a conversation, take turns, acknowledge each other's contributions, and establish common ground.

- World knowledge - issues which relate to external knowledge, which might be linguistic (e.g. cultural or pragmatic subtleties) or not 


\begin{tabular}{|c|c|c|c|c|c|}
\hline Turn & Role & Anonymised & Corrected & Resp.to & Sequence \\
\hline 1 & $\mathrm{~T}$ & $\begin{array}{l}\text { Hi there }\langle\text { STUDENT }\rangle \text {, all } \\
\text { OK? }\end{array}$ & $\begin{array}{l}\text { Hi there }\langle\text { STUDENT }\rangle \text {, all } \\
\text { OK? }\end{array}$ & & opening \\
\hline 2 & $\mathrm{~S}$ & $\begin{array}{l}\mathrm{Hi}\langle\mathrm{TEACHER}\rangle \text {, how are } \\
\text { you? }\end{array}$ & $\begin{array}{l}\text { Hi }\langle\text { TEACHER }\rangle \text {, how are } \\
\text { you? }\end{array}$ & & \\
\hline 3 & $\mathrm{~S}$ & $\begin{array}{l}\text { I did the exercise this } \\
\text { morning }\end{array}$ & $\begin{array}{l}\text { I did some exercise this } \\
\text { morning }\end{array}$ & & \\
\hline 4 & $\mathrm{~S}$ & I have done, I guess & I have done, I guess & & repair \\
\hline 5 & $\mathrm{~T}$ & $\begin{array}{l}\text { did is fine especially if } \\
\text { you're focusing on the } \\
\text { action itself }\end{array}$ & $\begin{array}{l}\text { did is fine especially if } \\
\text { you're focusing on the } \\
\text { action itself }\end{array}$ & & scaffolding \\
\hline 6 & $\mathrm{~T}$ & $\begin{array}{l}\text { tell me about your exercise } \\
\text { if you like! }\end{array}$ & $\begin{array}{l}\text { tell me about your exercise } \\
\text { if you like! }\end{array}$ & 3 & topic.dev \\
\hline
\end{tabular}

Table 1: Example of numbered, anonymised and annotated turns in the TSCC (where role T=teacher, S=student, and 'resp.to' means 'responding to'); the student is here chatting about physical exercise.

(they might simply be relevant to the current topic and task).

- Meta knowledge - discussion about the type of knowledge required for learning and assessment; for instance, 'there's been a shift to focus on $\mathrm{X}$ in teaching in recent years'.

- Typo - orthographic issues such as spelling, grammar or punctuation mistake

- Content - a repair sequence which involves a correction in meaning; for instance, Turn 1: Yes, that's fine. Turn 2: Oh wait, no, it's not correct.

- Exam practice - specific drills to prepare for examination scenarios.

- Admin - lesson management, such as 'please check your email' or 'see page 75 '.

Use of resource: At times the teacher refers the student to materials in support of learning. These can be the chat itself - where the teacher asks the student to review some previous turns in that same lesson - or a textbook page, online video, social media account, or other website.

Student assessment: The annotator, a qualified and experienced examiner of the English language, assessed the proficiency level shown by the student in each lesson. Assessment was applied according to the Common European Framework of Reference for Languages (CEFR) ${ }^{2}$, with levels from A1 (least advanced) to C2 (most advanced). We anticipated that students would get

\footnotetext{
${ }^{2}$ https: / / www. cambridgeenglish.org/ exams-and-tests/cefr
}

\begin{tabular}{l|rrr} 
Section & Lessons & Conv.turns & Words \\
\hline Teachers & 102 & 7632 & 93,602 \\
Students & 102 & 5920 & 39,293 \\
\hline All & 102 & 13,552 & 132,895
\end{tabular}

Table 2: Number of lessons, conversational turns and words in the TSCC contributed by teachers, students and all combined.

\begin{tabular}{l|rrr} 
Section & Lessons & Conv.turns & Words \\
\hline B1 & 36 & 1788 & 11,898 \\
B2 & 37 & 2394 & 11,331 \\
C1 & 29 & 1738 & 16,064 \\
\hline Students & 102 & 5920 & 39,293
\end{tabular}

Table 3: Number of lessons, conversational turns and words in the TSCC grouped by CEFR level.

more out of the lessons if they were already at a fairly good level, and therefore aimed our recruitment of participants at the intermediate level and above (CEFR B1 upwards). Assessment was applied in a holistic way based on the student's turns in each lesson: evaluating use of language (grammar and vocabulary), coherence, discourse management and interaction.

In Table 1 we exemplify many of the annotation steps described above with an excerpt from the corpus. We show several anonymised turns from one of the lessons, with turn numbers, participant role, error correction, 'responding to' when not the immediately preceding turn, and sequence type labels. Other labels such as teaching focus and use of resource are in the files but not shown 


\begin{tabular}{ll|r|r|r} 
& & FCE & CrowdED & TSCC \\
\hline \multirow{3}{*}{ Edit type } & Missing & $21.0 \%$ & $13.9 \%$ & $18.2 \%$ \\
& Replacement & $64.4 \%$ & $47.9 \%$ & $72.3 \%$ \\
& Unnecessary & $11.5 \%$ & $38.2 \%$ & $9.5 \%$ \\
\hline \multirow{6}{*}{ Adjective } & $1.4 \%$ & $0.8 \%$ & $1.5 \%$ \\
& Adjective:form & $0.3 \%$ & $0.06 \%$ & $0.1 \%$ \\
& Adverb & $1.9 \%$ & $1.5 \%$ & $1.6 \%$ \\
Conjunction & $0.7 \%$ & $1.3 \%$ & $0.2 \%$ \\
Contraction & $0.3 \%$ & $0.4 \%$ & $0.1 \%$ \\
Determiner & $10.9 \%$ & $4.0 \%$ & $12.4 \%$ \\
Morphology & $1.9 \%$ & $0.6 \%$ & $2.4 \%$ \\
Noun & $4.6 \%$ & $5.8 \%$ & $9.0 \%$ \\
Noun:inflection & $0.5 \%$ & $0.01 \%$ & $0.1 \%$ \\
Noun:number & $3.3 \%$ & $1.0 \%$ & $2.1 \%$ \\
Noun:possessive & $0.5 \%$ & $0.1 \%$ & $0.03 \%$ \\
Orthography & $2.9 \%$ & $3.0 \%$ & $6.7 \%$ \\
Other & $13.3 \%$ & $61.0 \%$ & $28.4 \%$ \\
Particle & $0.3 \%$ & $0.5 \%$ & $0.6 \%$ \\
& Preposition & $11.2 \%$ & $2.9 \%$ & $7.4 \%$ \\
Pronoun & $3.5 \%$ & $1.2 \%$ & $2.9 \%$ \\
Punctuation & $9.7 \%$ & $8.7 \%$ & $0.9 \%$ \\
Spelling & $9.6 \%$ & $0.3 \%$ & $6.0 \%$ \\
Verb & $7.0 \%$ & $3.1 \%$ & $6.7 \%$ \\
Verb:form & $3.6 \%$ & $0.4 \%$ & $2.9 \%$ \\
Verb:inflection & $0.2 \%$ & $0.01 \%$ & $0.1 \%$ \\
Verb:subj-verb-agr & $1.5 \%$ & $0.3 \%$ & $1.8 \%$ \\
Verb:tense & $6.0 \%$ & $1.1 \%$ & $4.8 \%$ \\
Word order & $1.8 \%$ & $1.2 \%$ & $1.0 \%$ \\
\hline Texts & 1244 & 1108 & 102 \\
Words & 531,416 & 39,726 & 132,895 \\
& Total edits & 52,671 & 8454 & 3800 \\
& & &
\end{tabular}

Table 4: The proportional distribution of error types determined by grammatical error correction of texts in the TSCC. Proportions supplied for the FCE Corpus for comparison, from Bryant et al. (2019), and a subset of the CROwDED Corpus (for a full description of error types see Bryant et al. (2017))

here. The example is not exactly how the corpus texts are formatted, but it serves to illustrate: the README distributed with the corpus further explains the contents of each annotated chat file.

The annotation of the features described above may in the long-term enable improved dialogue systems for language learning, and for the moment we view them as a first small step towards that larger goal. We do not yet know which features will be most useful and relevant for training such dialogue systems, but that is the purpose of collecting wide-ranging annotation. The corpus size is still relatively small, and so for the time being they allow us to focus on the analysis of one-toone chat lessons and understand how such lessons are structured by both teacher and student.

\section{Corpus analysis}

In Table 2 we report the overall statistics for TSCC in terms of lessons, conversational turns, and number of words (counted as white-space delimited tokens). We also show these statistics for the teacher and student groups separately. It is unsurprising that the teachers contribute many more turns and words to the chats than their students, but perhaps surprising just how much more they contribute. Each lesson was approximately one hour long and amounted to an average of 1300 words.

In Table 3 we show these same statistics for the student group only, and this time subsetting the 

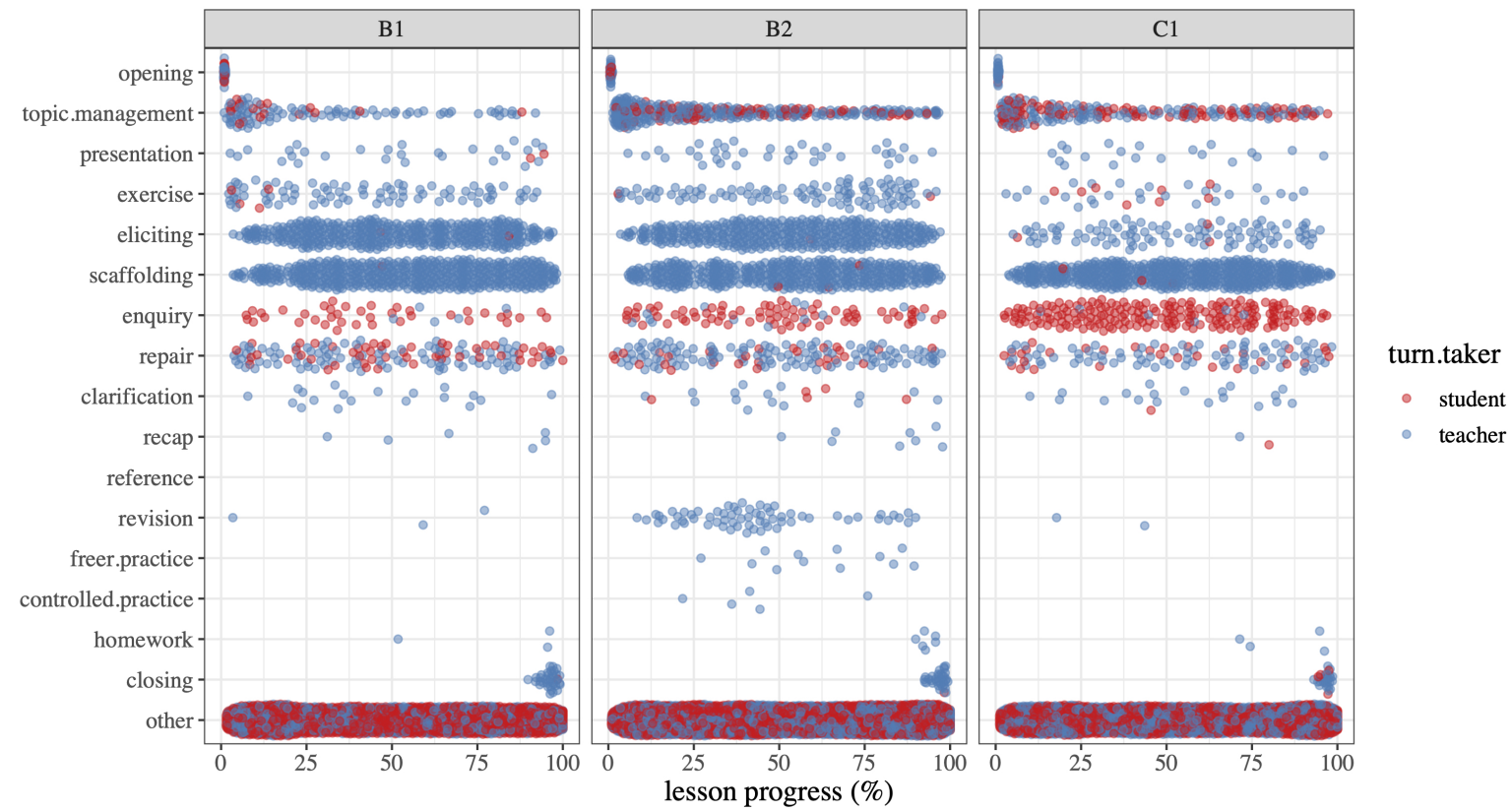

Figure 2: Selected sequence types in the TSCC, one plot per CEFR level, teachers as blue points, students as red; types on the $y$-axis and lesson progress on the $x$-axis (\%). 'Other' represents all non-sequence-starting turns in the corpus.

group by the CEFR levels found in the corpus: B1, $\mathrm{B} 2$ and $\mathrm{C} 1$. As expected, no students were deemed to be of CEFR level A1 or A2 in their written English, and the majority were of the intermediate $\mathrm{B} 1$ and B2 levels. It is notable that the B2 students in the corpus contribute many more turns than their $\mathrm{B} 1$ counterparts, but fewer words. The $\mathrm{C} 1$ students - the least numerous group - contribute the fewest turns of all groups but by far the most words. All the above might well be explained by individual variation and/or by teacher task and topic selection (e.g. setting tasks which do or do not invite longer responses) per the notion of 'opportunity of use' - what skills the students get the chance to demonstrate depends on the linguistic opportunities they are given (Caines and Buttery, 2017). Certainly we did find that student performance varied from lesson to lesson, so that the student might be B2 in one lesson for instance, and B1 or C1 in others. In future work, we wish to systematically examine the interplay between lesson structure, teaching feedback and student performance, because at present we can only observe that performance may vary from lesson to lesson.

The grammatical error correction performed on student turns in TSCC enables subsequent analysis of error types. We align each student turn with its corrected version, and then type the differences found according to the error taxonomy of Bryant et al. (2017) and using the ERRANT program ${ }^{3}$. We then count the number of instances of each error type and present them, following Bryant et al. (2019), as major edit types ('missing', 'replacement' and 'unnecessary' words) and grammatical error types which relate more to parts-of-speech and the written form. To show how TSCC compares to other error-annotated corpora, in Table 4 we present equivalent error statistics for the FCE Corpus of English exam essays at B1 or B2 level (Yannakoudakis et al., 2011) and the CROwDED Corpus of exam-like speech monologues by native and non-native speakers of English (Caines et al., 2016).

It is apparent in Table 4 that in terms of the distribution of edits and errors the TSCC is more alike to another written corpus, the FCE, than it is to a speech corpus (CROWDED). For instance, there are far fewer 'unnecessary' edit types in the TSCC than in CROWDED, with the majority being 'replacement' edit types like the FCE. For the error types, there is a smaller catch-all 'other' category for TSCC than CROWDED, along with many determiner, noun and preposition errors in common with FCE. There is a focus on the written form, with many orthography and spelling errors, but far fewer punctuation errors than the other cor-

\footnotetext{
${ }^{3}$ https://github.com/chrisjbryant/ errant
} 
pora - a sign that chat interaction has almost no standard regarding punctuation.

In Figure 2 we show where selected sequence types begin as points in the progress of each lesson (expressed as percentages) and which participant begins them, the teacher or student. Opening and closing sequences are where we might expect them at the beginning and end of lessons. The bulk of topic management occurs at the start of lessons and the bulk of eliciting and scaffolding occurs mid-lesson. Comparing the different CEFR levels, there are many fewer exercise and eliciting sequences for the $\mathrm{C} 1$ students compared to the B1 and B2 students; in contrast the C1 students do much more enquiry. In future work we aim to better analyse the scaffolding, repair and revision sequences in particular, to associate them with relevant preceding turns and understand what prompted the onset of these particular sequences.

\section{Conclusion}

We have described the Teacher-Student Chatroom Corpus, which we believe to be the first resource of its kind available for research use, potentially enabling both close discourse analysis and the eventual development of educational technology for practice in written English conversation. It currently contains 102 one-to-one lessons between two teachers and eight students of various ages and backgrounds, totalling $133 \mathrm{~K}$ words, along with annotation for a range of linguistic and pedagogic features. We demonstrated how such annotation enables new insight into the language teaching process, and propose that in future the dataset can be used to inform dialogue system design, in a similar way to Höhn's work with the Germanlanguage deL1L2IM corpus (Höhn, 2017).

One possible outcome of this work is to develop an engaging chatbot which is able to perform a limited number of language teaching tasks based on pedagogical expertise and insights gained from the TSCC. The intention is not to replace human teachers, but the chatbot can for example lighten the load of running a lesson - taking the 'easier' administrative tasks such as lesson opening and closing, or homework-setting - allowing the teacher to focus more on pedagogical aspects, or to multi-task across several lessons at once. This would be a kind of human-in-the-loop dialogue system or, from the teacher's perspective, assistive technology which can bridge between high quality but non-scalable one-to-one tutoring, and the current limitations of natural language processing technology. Such educational technology can bring the benefit of personalised tutoring, for instance reducing the anxiety of participating in group discussion (Griffin and Roy, 2019), while also providing the implicit skill and sensitivity brought by experienced human teachers.

First though, we need to demonstrate that (a) such a CALL system would be a welcome innovation for learners and teachers, and that (b) chatroom lessons do benefit language learners. We have seen preliminary evidence for both, but it remains anecdotal and a matter for thorough investigation in future. Collecting more data of the type described here will allow us to more comprehensively cover different teaching styles, demographic groups and L1 backgrounds. At the moment any attempt to look at individual variation can only be that: our group sizes are not yet large enough to be representative. We also aim to better understand the teaching actions contained in our corpus, how feedback sequences relate to the preceding student turns, and how the student responds to this feedback both within the lesson and across lessons over time.

\section{Acknowledgments}

This paper reports on research supported by Cambridge Assessment, University of Cambridge. Additional funding was provided by the Cambridge Language Sciences Research Incubator Fund and the Isaac Newton Trust. We thank Jane Walsh, Jane Durkin, Reka Fogarasi, Mark Brenchley, Mark Cresham, Kate Ellis, Tanya Hall, Carol Nightingale and Joy Rook for their support. We are grateful to the teachers, students and annotators without whose enthusiastic participation this corpus would not have been feasible.

\section{References}

Sherrie Atwood, William Turnbull, and Jeremy I. M. Carpendale. 2010. The construction of knowledge in classroom talk. Journal of the Learning Sciences, 19(3):358-402.

Douglas Biber, Susan Conrad, Randi Reppen, Pat Byrd, Marie Helt, Victoria Clark, Viviana Cortes, Eniko Csomay, and Alfredo Urzua. 2004. Representing language use in the university: Analysis of the TOEFL 2000 spoken and written academic language corpus. Princeton, NJ: Educational Testing Service. 
BNC Consortium. 2001. The British National Corpus, version 2 (BNC World).

Christopher Bryant, Mariano Felice, Øistein Andersen, and Ted Briscoe. 2019. The BEA-2019 Shared Task on Grammatical Error Correction. In Proceedings of the Fourteenth Workshop on Innovative Use of NLP for Building Educational Applications (BEA).

Christopher Bryant, Mariano Felice, and Ted Briscoe. 2017. Automatic annotation and evaluation of error types for grammatical error correction. In Proceedings of the 55th Annual Meeting of the Association for Computational Linguistics (Volume 1: Long Papers).

Christopher Bryant and Hwee Tou Ng. 2015. How far are we from fully automatic high quality grammatical error correction? In Proceedings of the 53rd Annual Meeting of the Association for Computational Linguistics and the 7th International Joint Conference on Natural Language Processing (Volume 1: Long Papers).

Paweł Budzianowski, Tsung-Hsien Wen, Bo-Hsiang Tseng, Iñigo Casanueva, Stefan Ultes, Osman Ramadan, and Milica Gašić. 2018. MultiWOZ - a large-scale multi-domain Wizard-of-Oz dataset for task-oriented dialogue modelling. In Proceedings of the 2018 Conference on Empirical Methods in Natural Language Processing.

Andrew Caines, Christian Bentz, Dimitrios Alikaniotis, Fridah Katushemererwe, and Paula Buttery. 2016. The glottolog data explorer: Mapping the world's languages. In Proceedings of the LREC 2016 Workshop 'VisLR II: Visualization as Added Value in the Development, Use and Evaluation of Language Resources'.

Andrew Caines and Paula Buttery. 2017. The effect of task and topic on language use in learner corpora. In Lynne Flowerdew and Vaclav Brezina, editors, Written and spoken learner corpora and their use in different contexts. London: Bloomsbury.

Ronald Carter and Michael McCarthy. 1997. Exploring Spoken English. Cambridge: Cambridge University Press.

Winston Chang, Joe Cheng, JJ Allaire, Yihui Xie, and Jonathan McPherson. 2020. shiny: Web Application Framework for R. R package version 1.4.0.2.

Eniko Csomay. 2012. Lexical Bundles in Discourse Structure: A Corpus-Based Study of Classroom Discourse. Applied Linguistics, 34(3):369-388.

Cristian Danescu-Niculescu-Mizil and Lillian Lee. 2011. Chameleons in imagined conversations: A new approach to understanding coordination of linguistic style in dialogs. In Proceedings of the 2 nd Workshop on Cognitive Modeling and Computational Linguistics.
Mihail Eric, Rahul Goel, Shachi Paul, Abhishek Sethi, Sanchit Agarwal, Shuyang Gao, Adarsh Kumar, Anuj Goyal, Peter Ku, and Dilek Hakkani-Tur. 2020. MultiWOZ 2.1: A consolidated multi-domain dialogue dataset with state corrections and state tracking baselines. In Proceedings of The 12th Language Resources and Evaluation Conference.

Jane Evison. 2013. Turn openings in academic talk: where goals and roles intersect. Classroom Discourse, 4(1):3-26.

Lynda Griffin and James Roy. 2019. A great resource that should be utilised more, but also a place of anxiety: student perspectives on using an online discussion forum. Open Learning: The Journal of Open, Distance and e-Learning, pages 1-16.

Sviatlana Höhn. 2017. A data-driven model of explanations for a chatbot that helps to practice conversation in a foreign language. In Proceedings of the 18th Annual SIGdial Meeting on Discourse and Dialogue.

Ian Hutchby and Robin Wooffitt. 1988. Conversation analysis. Cambridge: Polity Press.

Robbie Love, Claire Dembry, Andrew Hardie, Vaclav Brezina, and Tony McEnery. 2017. The Spoken BNC2014: designing and building a spoken corpus of everyday conversations. International Journal of Corpus Linguistics, 22:319-344.

Anne O'Keeffe and Steve Walsh. 2012. Applying corpus linguistics and conversation analysis in the investigation of small group teaching in higher education. Corpus Linguistics and Linguistic Theory, 8:159-181

Alan Ritter, Colin Cherry, and Bill Dolan. 2010. Unsupervised modeling of Twitter conversations. In Human Language Technologies: The 2010 Annual Conference of the North American Chapter of the Association for Computational Linguistics.

Stephen Roller, Emily Dinan, Naman Goyal, Da Ju, Mary Williamson, Yinhan Liu, Jing Xu, Myle Ott, Kurt Shuster, Eric M. Smith, Y-Lan Boureau, and Jason Weston. 2020. Recipes for building an opendomain chatbot. arXiv, 2004.13637.

Carolyn P. Rosé, Diane Litman, Dumisizwe Bhembe, Kate Forbes, Scott Silliman, Ramesh Srivastava, and Kurt VanLehn. 2003. A comparison of tutor and student behavior in speech versus text based tutoring. In Proceedings of the HLT-NAACL 03 Workshop on Building Educational Applications Using Natural Language Processing.

Harvey Sacks, Emanuel Schegloff, and Gail Jefferson. 1974. A simplest systematics for the organization of turn-taking for conversation. Language, 50:696735 . 
Nicolas Schrading, Cecilia Ovesdotter Alm, Ray Ptucha, and Christopher Homan. 2015. An analysis of domestic abuse discourse on Reddit. In Proceedings of the 2015 Conference on Empirical Methods in Natural Language Processing.

Paul Seedhouse. 2004. Conversation analysis methodology. Language Learning, 54:1-54.

Rita Simpson, Susan Briggs, John Ovens, and John Swales. 2002. Michigan Corpus of Academic Spoken English. Ann Arbor: University of Michigan.

Katherine Stasaski, Kimberly Kao, and Marti A. Hearst. 2020. CIMA: A large open access dialogue dataset for tutoring. In Proceedings of the Fifteenth Workshop on Innovative Use of NLP for Building Educational Applications.

Etsuko Toyoda and Richard Harrison. 2002. Categorization of text chat communication between learners and native speakers of Japanese. Language Learning Technology, 6:82-99.

Helen Yannakoudakis, Ted Briscoe, and Ben Medlock. 2011. A new dataset and method for automatically grading ESOL texts. In Proceedings of the 49th Annual Meeting of the Association for Computational Linguistics: Human Language Technologies. 